Supporting Information for Publication

\title{
On the Differences in the Mechanisms of
}

\section{Reduction of $\mathrm{AuCl}_{2}{ }^{-}$and $\mathrm{Ag}\left(\mathrm{H}_{2} \mathrm{O}\right)_{2}{ }^{+}$with $\mathrm{BH}_{4}{ }^{-}$}

Totan Mondal, ${ }^{1}$ Alina Sermiagin, ${ }^{1}$ Tomer Zidki, ${ }^{1}$ Allon Bogot, ${ }^{1}$ Dan Meyerstein

1,2 Haya Kornweitz*1

1 Chemical Sciences Department and The Radical Research Center, Ariel University, Ariel, Israel.

${ }^{2}$ Chemistry Department, Ben-Gurion University, Beer-Sheva, Israel. 
Cartesian coordinates of all relevant reactants, intermediates, and transition states:

\begin{tabular}{|c|c|c|c|}
\hline \multicolumn{4}{|c|}{$\mathrm{BH}_{4}^{-}$} \\
\hline B & -0.000408000 & 0.000070000 & 0.000000000 \\
\hline $\mathrm{H}$ & 0.620012000 & 0.356438000 & -1.010066000 \\
\hline $\mathrm{H}$ & 0.620018000 & 0.356453000 & 1.010058000 \\
\hline $\mathrm{H}$ & -1.123578000 & 0.518849000 & -0.000001000 \\
\hline $\mathrm{H}$ & -0.114411000 & -1.232088000 & 0.000009000 \\
\hline \multicolumn{4}{|c|}{$\mathrm{AuCl}_{4}^{-}$} \\
\hline $\mathrm{Au}$ & 0.000000000 & 0.000000000 & 0.000000000 \\
\hline $\mathrm{Cl}$ & 0.000000000 & 2.353137000 & 0.000000000 \\
\hline $\mathrm{Cl}$ & 2.353137000 & 0.000000000 & 0.000000000 \\
\hline $\mathrm{Cl}$ & 0.000000000 & -2.353137000 & 0.000000000 \\
\hline $\mathrm{Cl}$ & -2.353137000 & 0.000000000 & 0.000000000 \\
\hline \multicolumn{4}{|c|}{$\mathrm{AuCl}_{3}\left(\mathrm{BH}_{3}\right)^{2-}$} \\
\hline $\mathrm{Au}$ & 0.139222000 & 0.126643000 & 0.000452000 \\
\hline $\mathrm{Cl}$ & -1.331728000 & 2.017699000 & -0.000248000 \\
\hline $\mathrm{Cl}$ & -2.110562000 & -1.820849000 & -0.000741000 \\
\hline $\mathrm{Cl}$ & 1.806884000 & -1.598771000 & -0.000223000 \\
\hline B & 2.044281000 & 1.688063000 & -0.001833000 \\
\hline $\mathrm{H}$ & 1.485813000 & 2.746456000 & -0.002992000 \\
\hline $\mathrm{H}$ & 2.547872000 & 1.319311000 & -1.027572000 \\
\hline $\mathrm{H}$ & 2.548251000 & 1.321796000 & 1.024628000 \\
\hline \multicolumn{4}{|c|}{$\mathrm{AuCl}_{2}^{-}$} \\
\hline
\end{tabular}




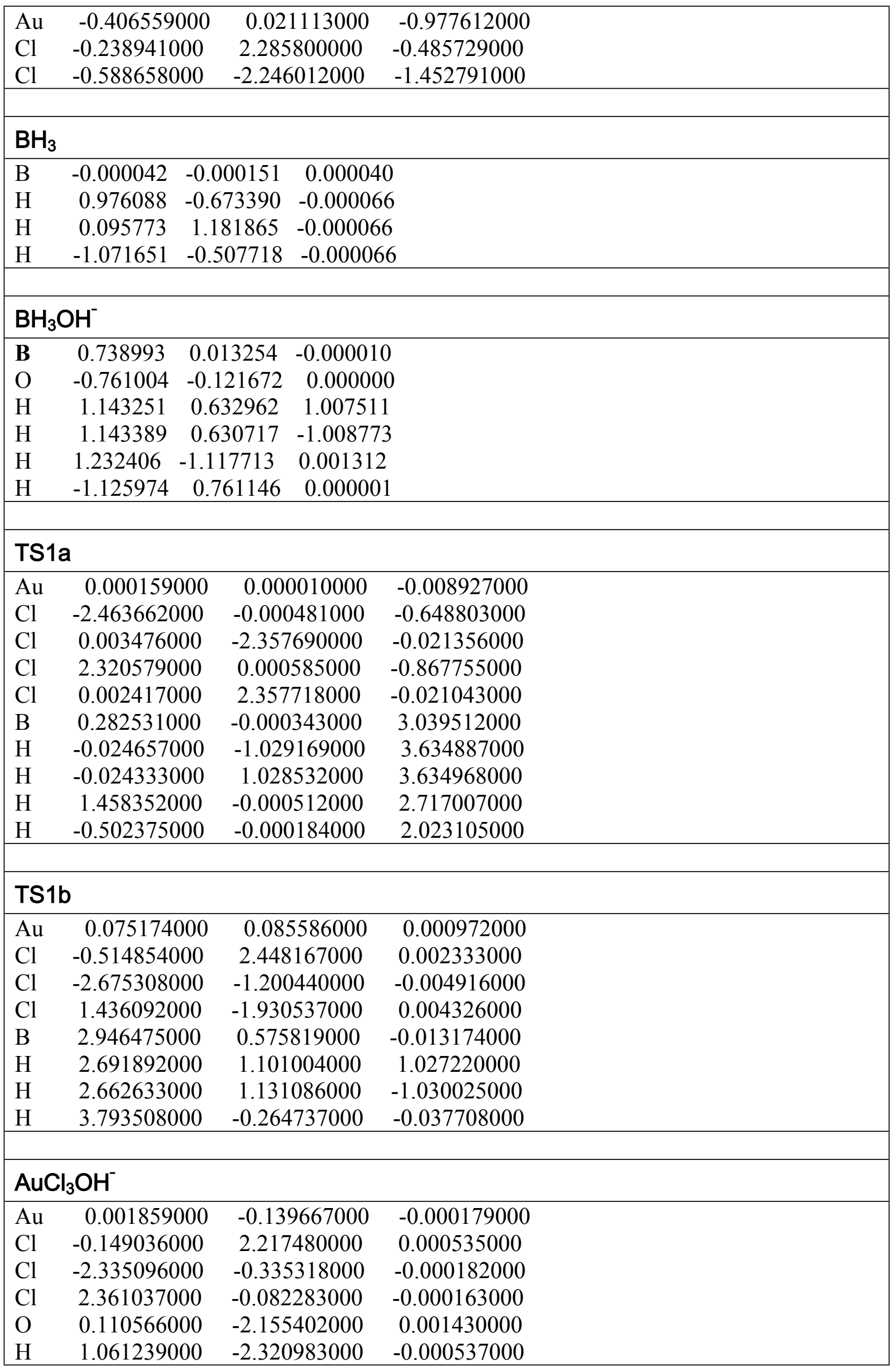




\begin{tabular}{|c|c|c|c|}
\hline \multicolumn{4}{|c|}{ TS1' } \\
\hline $\mathrm{Au}$ & 0.679255000 & -1.095612000 & 0.382487000 \\
\hline $\mathrm{Cl}$ & -1.034093000 & -1.877698000 & 1.879025000 \\
\hline $\mathrm{Cl}$ & 2.027794000 & -3.228951000 & 0.625897000 \\
\hline $\mathrm{Cl}$ & 2.258539000 & -0.093704000 & -1.143208000 \\
\hline $\mathrm{B}$ & -1.712580000 & 3.213389000 & -0.330553000 \\
\hline $\mathrm{H}$ & -1.783392000 & 3.855682000 & 0.707332000 \\
\hline $\mathrm{H}$ & -1.354634000 & 1.992045000 & 0.082421000 \\
\hline $\mathrm{H}$ & -2.786957000 & 3.023895000 & -0.882301000 \\
\hline $\mathrm{H}$ & -0.835691000 & 3.601097000 & -1.087076000 \\
\hline $\mathrm{O}$ & -0.440302000 & 0.722354000 & 0.159672000 \\
\hline $\mathrm{H}$ & 0.145428000 & 1.191233000 & -0.457396000 \\
\hline \multicolumn{4}{|c|}{ TS3 } \\
\hline $\mathrm{Au}$ & 0.004533000 & 0.071174000 & -0.000240000 \\
\hline $\mathrm{Cl}$ & -2.329487000 & -1.012547000 & 0.000418000 \\
\hline $\mathrm{Cl}$ & 2.288293000 & -1.061116000 & 0.000420000 \\
\hline $\mathrm{B}$ & 0.042744000 & 3.314984000 & 0.000650000 \\
\hline $\mathrm{H}$ & -1.090060000 & 3.786579000 & -0.001474000 \\
\hline $\mathrm{H}$ & 0.658030000 & 3.616549000 & 1.017146000 \\
\hline $\mathrm{H}$ & -0.103300000 & 2.030226000 & -0.003272000 \\
\hline $\mathrm{H}$ & 0.663821000 & 3.621263000 & -1.010967000 \\
\hline \multicolumn{4}{|c|}{$\mathrm{AuCl}\left(\mathrm{BH}_{4}\right)^{-}$} \\
\hline $\mathrm{Au}$ & 0.768103000 & -0.619482000 & 0.562688000 \\
\hline $\mathrm{Cl}$ & 1.817116000 & -0.887710000 & -1.543852000 \\
\hline $\mathrm{H}$ & -0.281403000 & -0.542553000 & 1.910981000 \\
\hline $\mathrm{B}$ & 0.489966000 & -0.019324000 & 2.907627000 \\
\hline $\mathrm{H}$ & 0.407603000 & 1.184999000 & 2.848383000 \\
\hline $\mathrm{H}$ & 1.621902000 & -0.443343000 & 3.000961000 \\
\hline $\mathrm{H}$ & -0.237073000 & -0.517761000 & 3.741802000 \\
\hline \multicolumn{4}{|c|}{ TS4 } \\
\hline $\mathrm{B}$ & -2.765296000 & 1.167494000 & -0.081306000 \\
\hline $\mathrm{H}$ & -1.143216000 & -1.452486000 & -0.152923000 \\
\hline $\mathrm{H}$ & -3.233434000 & 1.873660000 & 0.799573000 \\
\hline $\mathrm{H}$ & -3.023579000 & 1.556480000 & -1.204031000 \\
\hline $\mathrm{H}$ & -1.501258000 & 1.209468000 & 0.059956000 \\
\hline $\mathrm{O}$ & -3.231089000 & -0.263149000 & 0.083939000 \\
\hline $\mathrm{H}$ & -1.864579000 & -1.002253000 & -0.057878000 \\
\hline $\mathrm{H}$ & -3.516288000 & -0.382976000 & 0.989483000 \\
\hline $\mathrm{Au}$ & 0.157540000 & -0.169333000 & -0.027619000 \\
\hline $\mathrm{Cl}$ & 2.441877000 & 0.461362000 & 0.087221000 \\
\hline \multicolumn{4}{|c|}{ TS5 } \\
\hline $\mathrm{Au}$ & 0.193242000 & -0.062311000 & 0.033458000 \\
\hline
\end{tabular}




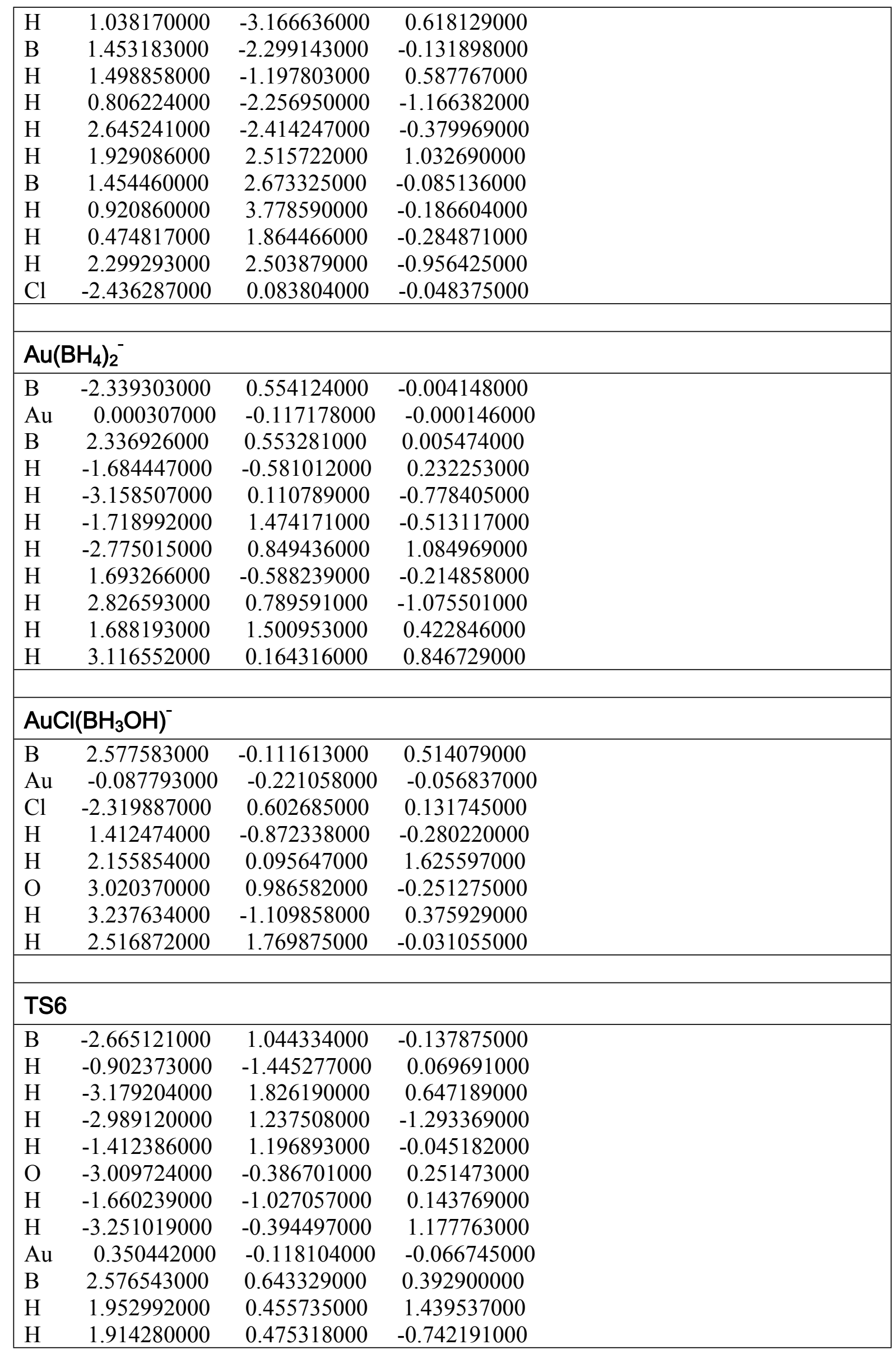




\begin{tabular}{|c|c|c|c|}
\hline $\mathrm{H}$ & 2.878821000 & 1.809410000 & 0.296893000 \\
\hline $\mathrm{H}$ & 3.483984000 & -0.148689000 & 0.291825000 \\
\hline \multicolumn{4}{|c|}{$\mathrm{Au}\left(\mathrm{BH}_{4}\right)\left(\mathrm{BH}_{3} \mathrm{OH}\right)^{-}$} \\
\hline B & -0.071789000 & 0.333998000 & 0.147714000 \\
\hline $\mathrm{Au}$ & -0.205958000 & -0.286970000 & 2.518870000 \\
\hline B & 1.379975000 & -0.148342000 & 4.673732000 \\
\hline $\mathrm{H}$ & -0.794690000 & -0.518747000 & 0.843563000 \\
\hline $\mathrm{H}$ & 0.478052000 & -0.403934000 & -0.639821000 \\
\hline $\mathrm{H}$ & 0.750233000 & 0.998269000 & 0.768377000 \\
\hline $\mathrm{H}$ & -0.928632000 & 1.043912000 & -0.330417000 \\
\hline $\mathrm{H}$ & -0.087007000 & -0.363974000 & 4.191367000 \\
\hline $\mathrm{H}$ & 1.058509000 & -0.624558000 & 5.734423000 \\
\hline $\mathrm{H}$ & 2.076884000 & -0.855280000 & 3.981389000 \\
\hline $\mathrm{O}$ & 1.679118000 & 1.233880000 & 4.729611000 \\
\hline $\mathrm{H}$ & 2.018255000 & 1.536704000 & 3.887226000 \\
\hline \multicolumn{4}{|c|}{$\mathrm{BH}(\mathrm{OH})_{2}$} \\
\hline $\mathrm{O}$ & 1.205477000 & -0.126124000 & -0.000187000 \\
\hline B & 0.000088000 & 0.500740000 & -0.000149000 \\
\hline $\mathrm{O}$ & -1.205477000 & -0.126101000 & 0.000259000 \\
\hline $\mathrm{H}$ & -0.000170000 & 1.684758000 & 0.000226000 \\
\hline $\mathrm{H}$ & 1.185209000 & -1.085327000 & 0.001783000 \\
\hline $\mathrm{H}$ & -1.185471000 & -1.085332000 & -0.001842000 \\
\hline \multicolumn{4}{|c|}{ TS7 } \\
\hline B & 2.551020000 & 0.403698000 & 0.617792000 \\
\hline $\mathrm{H}$ & 0.496366000 & -1.306501000 & -0.896488000 \\
\hline $\mathrm{H}$ & 3.178277000 & 0.572008000 & 1.662685000 \\
\hline $\mathrm{H}$ & 1.329078000 & 0.620312000 & 0.865811000 \\
\hline $\mathrm{O}$ & 2.625146000 & -1.015162000 & 0.140086000 \\
\hline $\mathrm{H}$ & 1.242780000 & -1.169371000 & -0.473908000 \\
\hline $\mathrm{H}$ & 3.313175000 & -1.030341000 & -0.530239000 \\
\hline $\mathrm{Au}$ & -0.602633000 & -0.167430000 & -0.022462000 \\
\hline B & -2.616063000 & 1.111929000 & 0.094243000 \\
\hline $\mathrm{H}$ & -1.960343000 & 1.401940000 & -0.909013000 \\
\hline $\mathrm{H}$ & -2.088028000 & 0.228146000 & 0.924505000 \\
\hline $\mathrm{H}$ & -2.704783000 & 2.073602000 & 0.820182000 \\
\hline $\mathrm{H}$ & -3.657631000 & 0.586094000 & -0.220669000 \\
\hline $\mathrm{O}$ & 3.060548000 & 1.213627000 & -0.495334000 \\
\hline $\mathrm{H}$ & 3.298806000 & 2.085261000 & -0.186561000 \\
\hline \multicolumn{4}{|c|}{$\mathrm{Au}\left(\mathrm{BH}_{4}\right) \mathrm{H}^{-}$} \\
\hline $\mathrm{Au}$ & 0.237172000 & -0.007255000 & -0.000169000 \\
\hline B & -2.291436000 & 0.048436000 & 0.001035000 \\
\hline $\mathrm{H}$ & -1.388180000 & -0.879481000 & 0.016441000 \\
\hline $\mathrm{H}$ & -2.948734000 & -0.237738000 & -0.982400000 \\
\hline $\mathrm{H}$ & -1.869440000 & 1.195205000 & -0.084105000 \\
\hline
\end{tabular}




\begin{tabular}{|c|c|c|c|}
\hline $\begin{array}{l}\mathrm{H} \\
\mathrm{H}\end{array}$ & $\begin{array}{c}-2.885251000 \\
1.812235000\end{array}$ & $\begin{array}{c}-0.115985000 \\
0.368991000\end{array}$ & $\begin{array}{l}1.050341000 \\
0.007872000\end{array}$ \\
\hline \multicolumn{4}{|c|}{ TS8 } \\
\hline B & 2.679838000 & 0.826249000 & 0.100622000 \\
\hline $\mathrm{H}$ & 0.551105000 & -1.273349000 & 0.871307000 \\
\hline $\mathrm{H}$ & 3.306890000 & 1.450732000 & -0.753022000 \\
\hline $\mathrm{H}$ & 3.025395000 & 1.126018000 & 1.234931000 \\
\hline $\mathrm{H}$ & 1.462522000 & 1.134417000 & -0.029332000 \\
\hline $\mathrm{O}$ & 2.853910000 & -0.666232000 & -0.081835000 \\
\hline $\mathrm{H}$ & 1.252089000 & -1.065817000 & 0.505675000 \\
\hline $\mathrm{H}$ & 2.873887000 & -0.847804000 & -1.021072000 \\
\hline $\mathrm{Au}$ & -0.591494000 & 0.000741000 & -0.001454000 \\
\hline $\mathrm{H}$ & -1.974300000 & 0.615879000 & -0.542083000 \\
\hline \multicolumn{4}{|c|}{$\mathrm{Au}\left(\mathrm{BH}_{3} \mathrm{OH}\right) \mathrm{H}^{-}$} \\
\hline B & -2.054660000 & 0.687862000 & 0.111937000 \\
\hline $\mathrm{Au}$ & 0.485640000 & -0.005495000 & -0.010234000 \\
\hline $\mathrm{H}$ & -0.967234000 & 0.754048000 & -0.722252000 \\
\hline $\mathrm{H}$ & -1.706928000 & 0.904495000 & 1.265932000 \\
\hline $\mathrm{O}$ & -2.788691000 & -0.547062000 & -0.095799000 \\
\hline $\mathrm{H}$ & -2.629636000 & 1.645343000 & -0.370002000 \\
\hline $\mathrm{H}$ & 1.890345000 & -0.682277000 & 0.443334000 \\
\hline $\mathrm{H}$ & -2.369272000 & -1.250328000 & 0.398175000 \\
\hline \multicolumn{4}{|c|}{ TS11 } \\
\hline $\mathrm{B}$ & -2.458417000 & -0.122700000 & 0.444391000 \\
\hline $\mathrm{H}$ & 0.087396000 & 1.620033000 & 0.660539000 \\
\hline $\mathrm{H}$ & -2.644744000 & 0.065278000 & 1.648461000 \\
\hline $\mathrm{H}$ & -1.341661000 & -0.693084000 & 0.281926000 \\
\hline $\mathrm{O}$ & -2.428499000 & 1.235312000 & -0.196692000 \\
\hline $\mathrm{H}$ & -0.640477000 & 1.469187000 & 0.352995000 \\
\hline $\mathrm{H}$ & -2.427863000 & 1.084404000 & -1.144983000 \\
\hline $\mathrm{Au}$ & 0.868704000 & -0.041626000 & -0.006370000 \\
\hline $\mathrm{H}$ & 2.078721000 & -0.996697000 & -0.447297000 \\
\hline $\mathrm{O}$ & -3.461532000 & -0.983969000 & -0.193835000 \\
\hline $\mathrm{H}$ & -4.326656000 & -0.657911000 & 0.053868000 \\
\hline \multicolumn{4}{|c|}{$\mathrm{AuH}_{2}{ }^{-}$} \\
\hline $\mathrm{Au}$ & 0.000007000 & -0.000008000 & -0.000005000 \\
\hline $\mathrm{H}$ & 1.110564000 & -0.653449000 & -1.089422000 \\
\hline $\mathrm{H}$ & -1.111148000 & 0.654074000 & 1.089826000 \\
\hline \multicolumn{4}{|c|}{ TS7' } \\
\hline B & -2.612763000 & 0.568385000 & 0.650858000 \\
\hline $\mathrm{H}$ & -0.777572000 & -1.324355000 & -0.949763000 \\
\hline $\mathrm{H}$ & -3.176961000 & 0.872695000 & 1.707677000 \\
\hline $\mathrm{H}$ & -1.366266000 & 0.746948000 & 0.828539000 \\
\hline
\end{tabular}




\begin{tabular}{|c|c|c|c|}
\hline $\mathrm{O}$ & -2.883774000 & -0.892064000 & 0.371706000 \\
\hline $\mathrm{H}$ & -1.417713000 & -1.163067000 & -0.427385000 \\
\hline $\mathrm{H}$ & -2.953459000 & -1.355000000 & 1.207716000 \\
\hline $\mathrm{Au}$ & 0.379632000 & -0.192659000 & -0.114939000 \\
\hline $\mathrm{O}$ & -2.990529000 & 1.325498000 & -0.522973000 \\
\hline $\mathrm{H}$ & -3.895908000 & 1.105067000 & -0.743029000 \\
\hline $\mathrm{Cl}$ & 2.567950000 & 0.589905000 & 0.318368000 \\
\hline \multicolumn{4}{|c|}{$\mathrm{AuClH}^{-}$} \\
\hline $\mathrm{Au}$ & 0.000191000 & -0.412080000 & 0.000000000 \\
\hline $\mathrm{Cl}$ & 0.000191000 & 2.033667000 & 0.000000000 \\
\hline $\mathrm{H}$ & -0.018346000 & -2.018006000 & 0.000000000 \\
\hline \multicolumn{4}{|c|}{ TS3' } \\
\hline $\mathrm{Au}$ & -0.241855000 & -0.400260000 & 0.000012000 \\
\hline $\mathrm{Cl}$ & 2.318708000 & 0.836303000 & -0.000018000 \\
\hline $\mathrm{H}$ & -0.741007000 & -1.954957000 & -0.000520000 \\
\hline B & -2.187067000 & 2.151271000 & -0.000049000 \\
\hline $\mathrm{H}$ & -2.814641000 & 1.833075000 & 1.010843000 \\
\hline $\mathrm{H}$ & -1.949616000 & 3.363871000 & 0.000061000 \\
\hline $\mathrm{H}$ & -1.058260000 & 1.572123000 & 0.001307000 \\
\hline $\mathrm{H}$ & -2.812659000 & 1.832908000 & -1.012090000 \\
\hline \multicolumn{4}{|c|}{ TS9 } \\
\hline $\mathrm{B}$ & -3.141876000 & 0.869237000 & -0.163139000 \\
\hline $\mathrm{H}$ & -1.050873000 & -1.395534000 & -0.468738000 \\
\hline $\mathrm{H}$ & -3.792070000 & 1.383197000 & 0.738558000 \\
\hline $\mathrm{H}$ & -3.447291000 & 1.292411000 & -1.264590000 \\
\hline $\mathrm{H}$ & -1.928681000 & 1.140649000 & 0.041185000 \\
\hline $\mathrm{O}$ & -3.317597000 & -0.642929000 & -0.138298000 \\
\hline $\mathrm{H}$ & -1.828750000 & -1.085560000 & -0.316058000 \\
\hline $\mathrm{H}$ & -3.589179000 & -0.896400000 & 0.743398000 \\
\hline $\mathrm{Au}$ & 0.060107000 & -0.005831000 & 0.038789000 \\
\hline $\mathrm{B}$ & 2.878638000 & 0.018718000 & 0.589912000 \\
\hline $\mathrm{H}$ & 2.634185000 & -1.014460000 & 1.142962000 \\
\hline $\mathrm{H}$ & 1.432239000 & 0.838930000 & 0.413432000 \\
\hline $\mathrm{H}$ & 3.190997000 & 0.956892000 & 1.284839000 \\
\hline $\mathrm{O}$ & 3.470382000 & -0.101234000 & -0.673689000 \\
\hline $\mathrm{H}$ & 3.724890000 & 0.754073000 & -1.017243000 \\
\hline \multicolumn{4}{|c|}{$\mathrm{Au}\left(\mathrm{BH}_{3} \mathrm{OH}\right)_{2}^{-}$} \\
\hline $\mathrm{O}$ & -2.944233000 & 1.106210000 & -0.482729000 \\
\hline B & -2.290740000 & 0.702963000 & 0.718300000 \\
\hline $\mathrm{Au}$ & 0.005623000 & -0.391775000 & -0.033476000 \\
\hline $\mathrm{B}$ & 2.592245000 & 0.261187000 & 0.139191000 \\
\hline $\mathrm{H}$ & 1.556147000 & -0.399432000 & -0.731420000 \\
\hline $\mathrm{H}$ & 2.419770000 & -0.134774000 & 1.272548000 \\
\hline $\mathrm{O}$ & 2.584553000 & 1.667534000 & -0.072564000 \\
\hline $\mathrm{H}$ & 3.461500000 & -0.318755000 & -0.466783000 \\
\hline
\end{tabular}




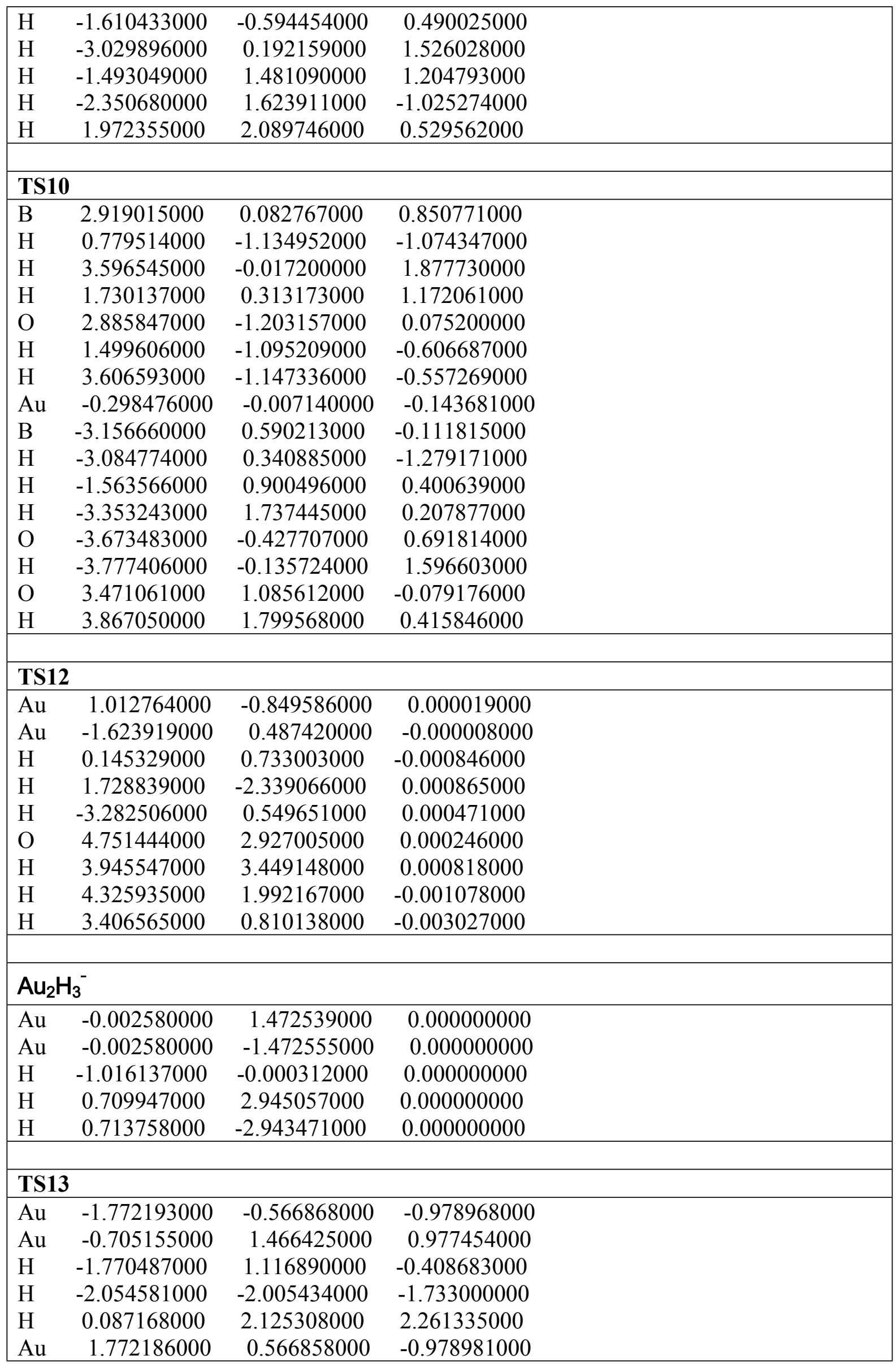




\begin{tabular}{|c|c|c|c|}
\hline $\mathrm{Au}$ & 0.705162000 & -1.466415000 & 0.977465000 \\
\hline $\mathrm{H}$ & 2.054631000 & 2.005446000 & -1.732945000 \\
\hline $\mathrm{H}$ & -0.087095000 & -2.125234000 & 2.261421000 \\
\hline $\mathrm{H}$ & 1.770380000 & -1.116928000 & -0.408771000 \\
\hline \multicolumn{4}{|c|}{$\mathrm{Au}_{4} \mathrm{H}_{6}{ }^{2-}$} \\
\hline $\mathrm{Au}$ & -1.209202000 & -1.061504000 & 1.103609000 \\
\hline $\mathrm{Au}$ & -1.264486000 & 1.009386000 & -1.104235000 \\
\hline $\mathrm{H}$ & -2.157732000 & -0.166523000 & -0.107048000 \\
\hline $\mathrm{H}$ & -0.683542000 & -2.042623000 & 2.317740000 \\
\hline $\mathrm{H}$ & -0.796862000 & 2.172244000 & -2.170431000 \\
\hline $\mathrm{Au}$ & 1.267738000 & -1.084517000 & -1.029156000 \\
\hline $\mathrm{Au}$ & 1.205945000 & 1.136116000 & 1.029380000 \\
\hline $\mathrm{H}$ & 0.802251000 & -2.238538000 & -2.108812000 \\
\hline $\mathrm{H}$ & 0.664067000 & 2.289115000 & 2.073101000 \\
\hline $\mathrm{H}$ & 2.172234000 & 0.027353000 & 0.027169000 \\
\hline \multicolumn{4}{|c|}{$\mathrm{Au}_{6} \mathrm{H}_{8}{ }^{2-}$} \\
\hline $\mathrm{Au}$ & -0.235904000 & -1.084076000 & -0.382902000 \\
\hline $\mathrm{Au}$ & 1.329566000 & 1.495535000 & -1.301093000 \\
\hline $\mathrm{Au}$ & 1.173453000 & 0.865707000 & 1.613577000 \\
\hline $\mathrm{Au}$ & 2.577895000 & -1.673790000 & -0.026570000 \\
\hline $\mathrm{H}$ & 0.920607000 & -2.370716000 & -0.368205000 \\
\hline $\mathrm{H}$ & -1.753691000 & -0.173532000 & -0.779957000 \\
\hline $\mathrm{H}$ & 4.186786000 & -1.520964000 & 0.217024000 \\
\hline $\mathrm{H}$ & 0.620388000 & 0.144117000 & 2.976077000 \\
\hline $\mathrm{H}$ & 0.953789000 & 1.401611000 & -2.894721000 \\
\hline $\mathrm{H}$ & 1.958235000 & 1.837758000 & 0.334117000 \\
\hline $\mathrm{Au}$ & -1.662371000 & 1.511665000 & 0.080279000 \\
\hline $\mathrm{Au}$ & -3.187984000 & -1.120574000 & 0.010821000 \\
\hline $\mathrm{H}$ & -1.933430000 & 3.052462000 & 0.534361000 \\
\hline $\mathrm{H}$ & -4.530437000 & -1.933695000 & 0.446452000 \\
\hline \multicolumn{4}{|c|}{$\mathrm{Au}_{6} \mathrm{H}_{6}{ }^{2-}$} \\
\hline $\mathrm{Au}$ & -1.392189000 & -1.195508000 & -0.873669000 \\
\hline $\mathrm{Au}$ & 3.222384000 & 0.977537000 & -0.302160000 \\
\hline $\mathrm{Au}$ & -0.097841000 & -0.850305000 & 2.010569000 \\
\hline $\mathrm{Au}$ & 1.426962000 & -1.223989000 & -0.784489000 \\
\hline $\mathrm{H}$ & 0.024064000 & -2.224875000 & -1.286198000 \\
\hline $\mathrm{H}$ & -3.076457000 & -0.776862000 & -0.849885000 \\
\hline $\mathrm{H}$ & 3.112546000 & -0.838224000 & -0.630452000 \\
\hline $\mathrm{H}$ & -0.162628000 & -1.979507000 & 3.181424000 \\
\hline $\mathrm{H}$ & 3.845995000 & 2.473118000 & -0.074766000 \\
\hline $\mathrm{Au}$ & 0.019420000 & 1.303144000 & 0.282238000 \\
\hline $\mathrm{Au}$ & -3.178205000 & 1.000097000 & -0.336865000 \\
\hline $\mathrm{H}$ & -3.785454000 & 2.479248000 & 0.005583000 \\
\hline
\end{tabular}




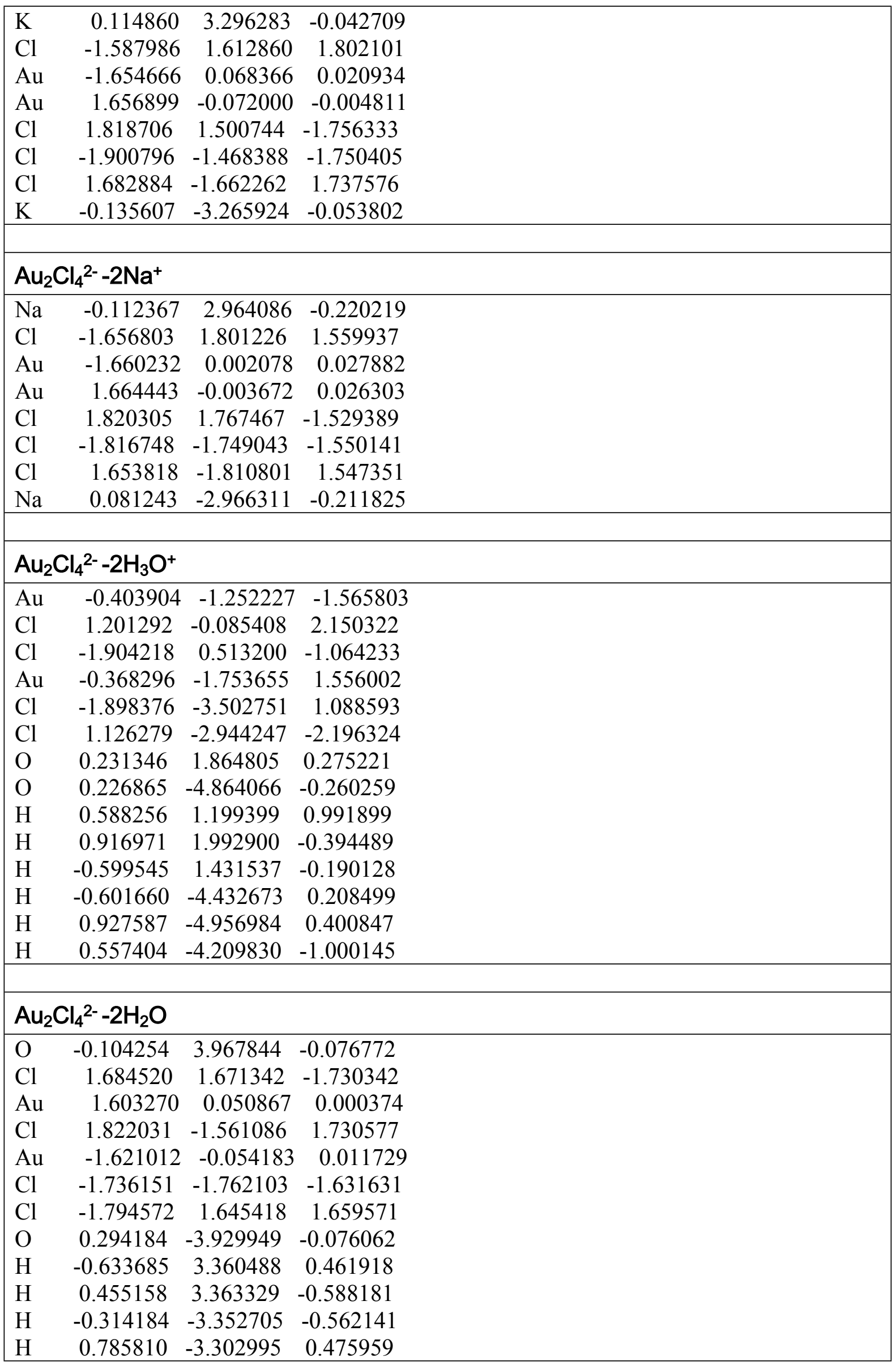




\begin{tabular}{|c|c|}
\hline \multicolumn{2}{|c|}{$\mathrm{Au}_{2} \mathrm{Cl}_{2}\left(\mathrm{BH}_{4}\right)_{2}{ }^{2-}-2 \mathrm{Na}^{+}$} \\
\hline $\mathrm{Au}$ & $\begin{array}{llll}1.703605 & 0.063406 & -0.175164\end{array}$ \\
\hline $\mathrm{Cl}$ & $\begin{array}{lll}1.602647 & -1.401445 & 1.698648\end{array}$ \\
\hline B & $\begin{array}{lll}1.830769 & 2.074133 & -1.516879\end{array}$ \\
\hline $\mathrm{Au}$ & $\begin{array}{lll}-1.670964 & 0.084957 & 0.114387\end{array}$ \\
\hline $\mathrm{Cl}$ & $-1.627788 \quad-1.625240 \quad-1.537272$ \\
\hline B & $\begin{array}{lll}-2.178881 & 2.173924 & 1.247579\end{array}$ \\
\hline $\mathrm{Na}$ & $-0.030080 \quad-2.826510 \quad 0.183597$ \\
\hline $\mathrm{Na}$ & $0.087000 \quad 3.011255 \quad 0.225719$ \\
\hline $\mathrm{H}$ & $\begin{array}{lll}2.621394 & 2.331879 & -2.379639\end{array}$ \\
\hline $\mathrm{H}$ & $\begin{array}{lll}0.695503 & 2.387173 & -1.808677\end{array}$ \\
\hline $\mathrm{H}$ & $\begin{array}{lll}1.892567 & 0.757791 & -1.740932\end{array}$ \\
\hline $\mathrm{H}$ & $2.236232 \quad 2.513250 \quad-0.450883$ \\
\hline $\mathrm{H}$ & $\begin{array}{lll}-2.187931 & 2.504483 & 0.072473\end{array}$ \\
\hline $\mathrm{H}$ & $\begin{array}{lll}-3.272828 & 2.161426 & 1.723760\end{array}$ \\
\hline $\mathrm{H}$ & $\begin{array}{lll}-1.377913 & 2.819494 & 1.900279\end{array}$ \\
\hline $\mathrm{H}$ & $\begin{array}{lll}-1.643760 & 0.985007 & 1.585571 \\
\end{array}$ \\
\hline \multicolumn{2}{|c|}{$\mathrm{Au}_{2} \mathrm{Cl}_{3}\left(\mathrm{BH}_{4}\right)^{2-}-2 \mathrm{Na}^{+}$} \\
\hline $\mathrm{Au}$ & $\begin{array}{lll}1.768549 & 0.029313 & -0.217739\end{array}$ \\
\hline $\mathrm{Cl}$ & $\begin{array}{lll}1.770902 & -1.149733 & 1.848038\end{array}$ \\
\hline B & $\begin{array}{lll}1.850149 & 1.901011 & -1.755667\end{array}$ \\
\hline $\mathrm{Au}$ & $\begin{array}{lll}-1.658915 & -0.003380 & -0.078525\end{array}$ \\
\hline $\mathrm{Cl}$ & $-1.523367 \quad-2.017379 \quad-1.303060$ \\
\hline $\mathrm{Cl}$ & $\begin{array}{lll}-1.903028 & 1.949390 & 1.221587\end{array}$ \\
\hline $\mathrm{Na}$ & $\begin{array}{lll}-0.028037 & -2.737633 & 0.749500\end{array}$ \\
\hline $\mathrm{Na}$ & $0.277968 \quad 2.909466 \quad 0.110674$ \\
\hline $\mathrm{H}$ & $\begin{array}{lll}2.564310 & 2.038915 & -2.707330\end{array}$ \\
\hline $\mathrm{H}$ & $\begin{array}{lll}0.703668 & 2.233246 & -1.972600\end{array}$ \\
\hline $\mathrm{H}$ & $\begin{array}{lll}1.849801 & 0.561208 & -1.851769\end{array}$ \\
\hline $\mathrm{H}$ & $\begin{array}{lll}2.364535 & 2.423999 & -0.778577 \\
\end{array}$ \\
\hline \multicolumn{2}{|c|}{$\mathrm{Au}_{2}\left(\mathrm{BH}_{4}\right)_{4}{ }^{2-}-2 \mathrm{Na}^{+}$} \\
\hline $\mathrm{B}$ & $\begin{array}{lll}-1.681675 & 1.793347 & 1.662817\end{array}$ \\
\hline $\mathrm{Au}$ & $\begin{array}{lll}-1.651100 & -0.017307 & 0.011522\end{array}$ \\
\hline $\mathrm{Au}$ & $\begin{array}{lll}1.652787 & 0.015934 & 0.008252\end{array}$ \\
\hline B & $\begin{array}{lll}1.676208 & 1.784020 & -1.686304\end{array}$ \\
\hline $\mathrm{B}$ & $\begin{array}{lll}-1.716157 & -1.813452 & -1.656792\end{array}$ \\
\hline $\mathrm{B}$ & $\begin{array}{lll}1.682517 & -1.778616 & 1.678663\end{array}$ \\
\hline $\mathrm{Na}$ & $\begin{array}{lll}-0.069359 & 2.980859 & -0.072667\end{array}$ \\
\hline $\mathrm{Na}$ & $\begin{array}{lll}0.086926 & -2.959514 & -0.074405\end{array}$ \\
\hline $\mathrm{H}$ & $\begin{array}{lll}-2.412934 & 1.941984 & 2.600272\end{array}$ \\
\hline $\mathrm{H}$ & $\begin{array}{lll}-0.532376 & 2.103863 & 1.909268\end{array}$ \\
\hline $\mathrm{H}$ & $\begin{array}{lll}-1.690219 & 0.471395 & 1.699023\end{array}$ \\
\hline $\mathrm{H}$ & $\begin{array}{lll}-2.160030 & 2.347946 & 0.682543\end{array}$ \\
\hline $\mathrm{H}$ & $\begin{array}{llll}2.084601 & 2.342154 & -0.675227\end{array}$ \\
\hline $\mathrm{H}$ & $\begin{array}{lll}2.475562 & 1.918238 & -2.567708 \\
\end{array}$ \\
\hline
\end{tabular}




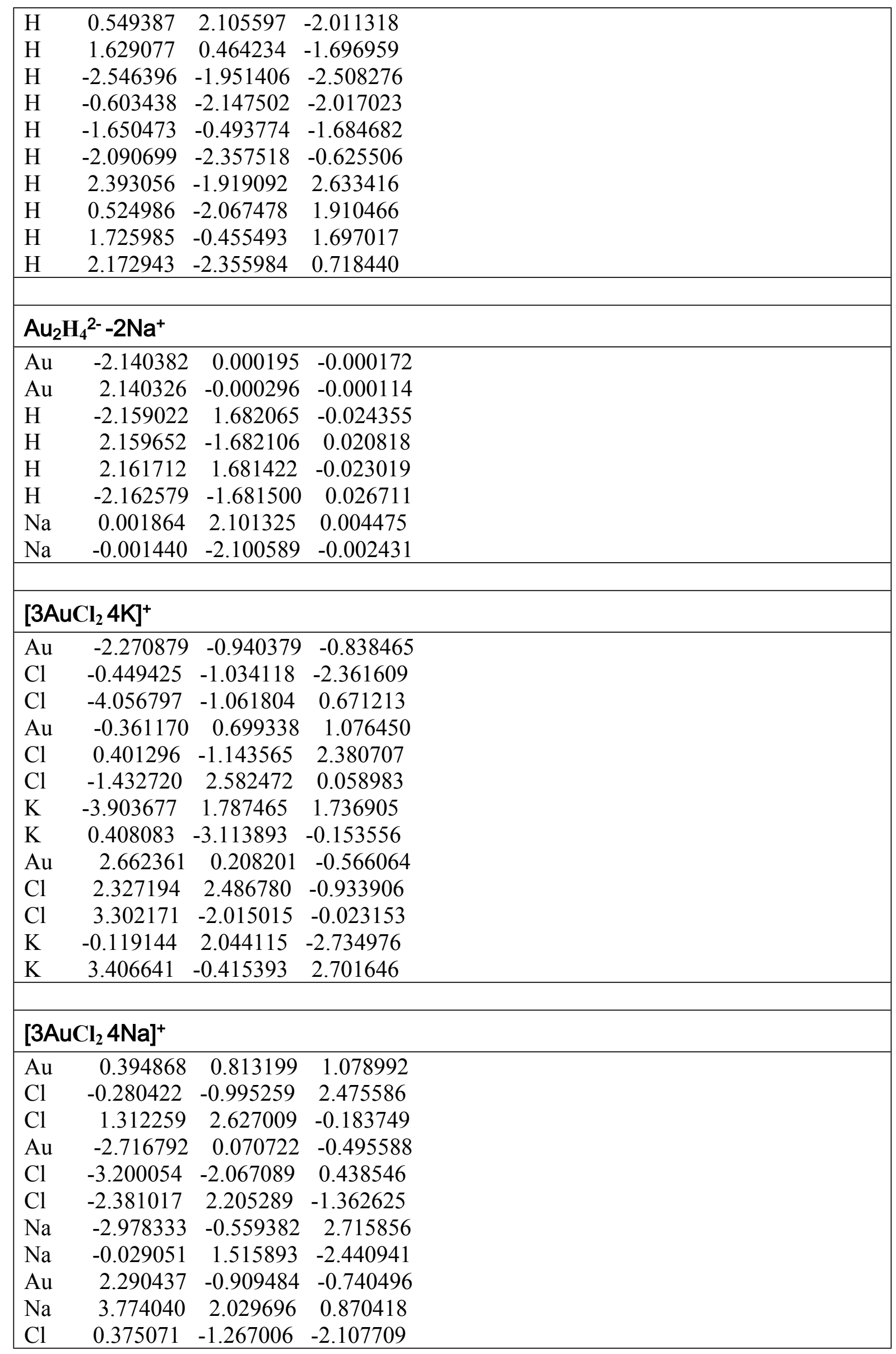




\begin{tabular}{|lccc|}
\hline $\mathrm{Cl}$ & 4.157969 & -0.585072 & 0.635050 \\
$\mathrm{Na}$ & -0.515495 & -2.675689 & 0.144992 \\
\hline \multicolumn{5}{|c|}{} \\
\hline [3AuCl$\left._{2} \mathbf{4 H}_{3} \mathrm{O}\right]^{+}$ & & \\
\hline $\mathrm{O}$ & -0.012634 & -2.953214 & -1.458637 \\
$\mathrm{Cl}$ & 0.008816 & -0.268581 & -2.498019 \\
$\mathrm{Au}$ & 0.006296 & 0.763521 & -0.373422 \\
$\mathrm{Cl}$ & 0.018723 & 1.915871 & 1.744988 \\
$\mathrm{Au}$ & 2.812367 & -0.460682 & 0.028779 \\
$\mathrm{Cl}$ & 3.751273 & 1.555564 & -0.783645 \\
$\mathrm{Cl}$ & 2.044493 & -2.589221 & 0.809765 \\
$\mathrm{O}$ & 2.619133 & 3.311558 & 1.051462 \\
$\mathrm{H}$ & 0.775534 & -2.993216 & -0.856390 \\
$\mathrm{H}$ & -0.810896 & -2.990660 & -0.869174 \\
$\mathrm{H}$ & -0.006557 & -2.019304 & -1.919834 \\
$\mathrm{H}$ & 3.096159 & 2.621202 & 0.313375 \\
$\mathrm{H}$ & 3.199440 & 3.504023 & 1.800469 \\
$\mathrm{H}$ & 1.754556 & 2.931741 & 1.369260 \\
$\mathrm{Au}$ & -2.813787 & -0.442516 & 0.004051 \\
$\mathrm{Cl}$ & -3.684486 & 1.611269 & -0.789555 \\
$\mathrm{Cl}$ & -2.102948 & -2.593258 & 0.775775 \\
$\mathrm{O}$ & -2.711203 & 3.171713 & 1.298967 \\
$\mathrm{H}$ & -1.782503 & 2.874694 & 1.501533 \\
$\mathrm{H}$ & -3.130813 & 2.572894 & 0.456777 \\
$\mathrm{H}$ & -2.734767 & 4.122681 & 1.125950 \\
$\mathrm{O}$ & -0.047163 & -1.876369 & 2.705439 \\
$\mathrm{H}$ & 0.780976 & -2.089393 & 2.139878 \\
$\mathrm{H}$ & -0.865636 & -2.089130 & 2.124663 \\
$\mathrm{H}$ & -0.055531 & -2.378511 & 3.534164 \\
\hline
\end{tabular}

